\title{
EHRA/ESC Left atrial appendage closure (LAAC) candidates at discharge after a stroke associated with atrial fibrillation (AF): 12 months outcome in the WATCH-AF registry
}

E. Ong ${ }^{1}$, E. Meseguer ${ }^{2}$, C. Guidoux², P. Lavallée ${ }^{2}$, C. Hobeanu' ${ }^{2}$, L.Monteiro-Tavares², L. Cabrejo ${ }^{2}$, A. Martins-Brechet ${ }^{2}$, R. Rigual ${ }^{2}$, N. Nighoghossian ${ }^{1}$, P. Amarenco ${ }^{2}$

1 Hospices Civils de Lyon, Neurology Department, Lyon, France 2 Bichat Universitary Hospital, Neurology Department, Paris, France

\section{Introduction}

Because of frailty, cognitive impairment, co-morbidities, patient refusal, or perceived low risk of stroke or high bleeding risk, many patients with stroke and AF cannot be discharged on long-term oral anticoagulant (LTOAC). Among them, the proportion of candidate for LAAC and their 12-month outcome is not well known.

\section{Methods}

Consecutive patients admitted within 24 hours of symptom onset of an acute stroke associated with AF in two stroke centers had systematic data collection (e.g., CHADSVASC, HASBLED) at 6 and 1 months before stroke, at admission, discharge, 2 and 8 days, 3, 12 months post stroke. The primary endpoint was death or dependency (mRS $>3$ ). Potential candidates for LAAC were based on ERHA/ESC recommendations (table).

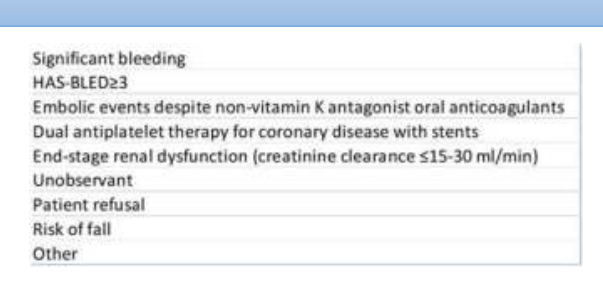

Table Reasons for performing LAAC (ERHA/ESC recommendations)

\section{Results}

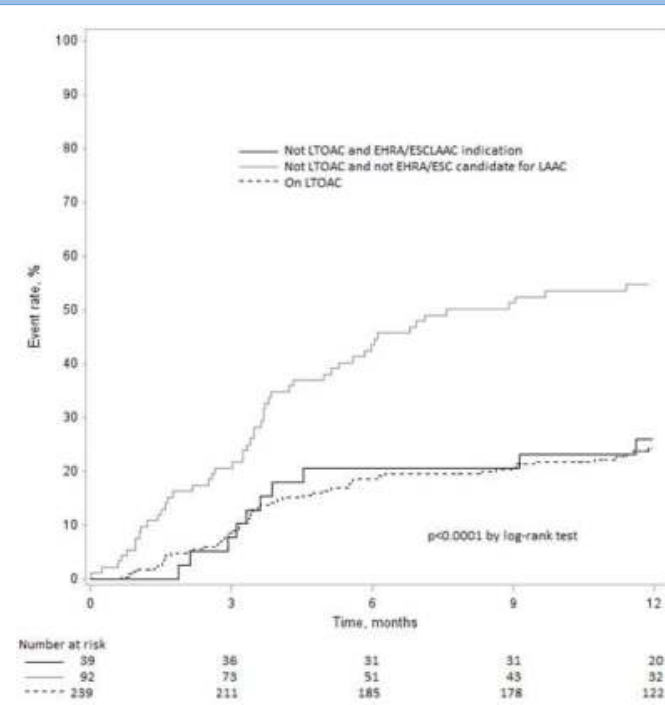

Figure 1 Cumulative event rate of 12-month risk of death and dependency according to LTOAC and LAAC indication

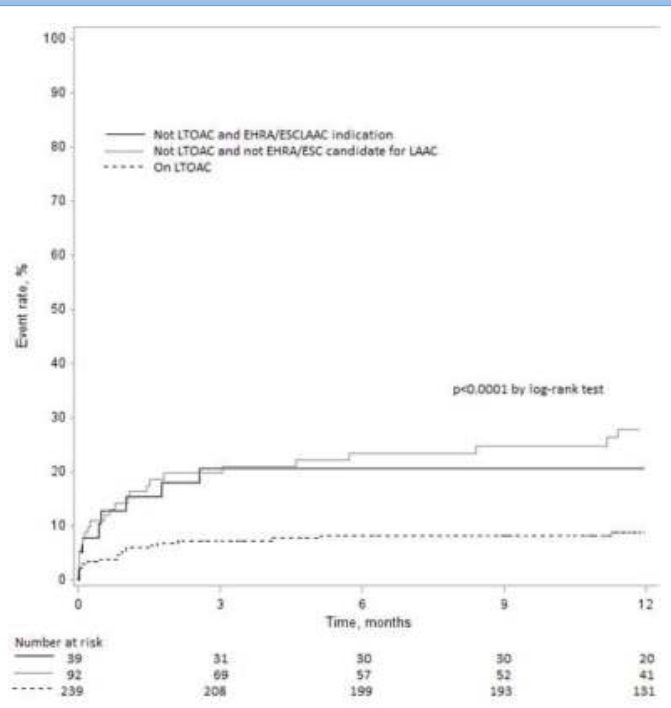

Figure 2 Cumulative event rate of 12 -month risk of brain infarction brain hemorrhage according to LTOAC and LAAC indication

$\Rightarrow \quad$ Among 400 enrolled patients, 30 patients died before discharge. Among 370 patients alive at discharge, 131 patients were not discharged on LTOAC, including 39/370 (10.5\%) who were EHRA/ESC LAAC indication.

$\Rightarrow \quad$ At 12 months, death or dependency occurred in $24.3 \%$ in 239 patients discharged on LTOAC, as compared to $54.7 \%$ in patients not discharged on LTOAC and not ERHA/ESC candidate for LAAC, and to $25.9 \%$ in patients not discharged on LTOAC and with ERHA/ESC LAAC indication $(p<0.001)$ (Figure 1).

$\Rightarrow \quad$ At 12 months, brain infarction brain hemorrhage occurred in $8.7 \%$ of patients discharged on LTOAC, as compared to $27.8 \%$ of patients not discharged on LTOAC and not EHRA/ESC candidate for LAAC and to $20.5 \%$ in patients not discharged on LTOAC and with EHRA/ESC LAAC indication $(p<0.001)$ (Figure 2$)$.

\section{Conclusion}

10.5\% of patients with stroke associated with atrial fibrillation were EHRA/ESC candidate for LAAC.

Their 12-month brain infarction brain hemorrhage risk was twice the risk of patients discharged on LTOAC.

Kirchhof P, Benussi S, Kotecha D, Ahlsson A, Atar D, Casadei B, Castella M, Diener HC, Heidbuchel H, Hendriks J, Hindricks G, Manolis AS, Oldgren J, Popescu BA, Schotten U, Van Putte B, Vardas P; ESC Scientific Document Group . 2016 ESC Guidelines for the management of atrial fibrillation developed in collaboration with EACTS. Eur Heart J. 2016 Oct 7;37(38):2893-2962 